\title{
On the functional equation defined by Lie's product formula
}

\author{
by \\ Gerd Herzog and Christoph Schmoeger (Karlsruhe)
}

\begin{abstract}
Let $E$ be a real normed space and $\mathcal{A}$ a complex Banach algebra with unit. We characterize the continuous solutions $f: E \rightarrow \mathcal{A}$ of the functional equation $f(x+y)=\lim _{n \rightarrow \infty}(f(x / n) f(y / n))^{n}$.
\end{abstract}

Let $\mathcal{A}$ be a complex Banach algebra with unit 1. In this setting the famous Lie product formula reads

$$
\exp (a+b)=\lim _{n \rightarrow \infty}(\exp (a / n) \exp (b / n))^{n} \quad(a, b \in \mathcal{A}) ;
$$

see [4, Theorem VIII.29] for matrices and a proof which also holds for Banach algebras, and Trotter's version for semigroups [6].

Let $p \in \mathcal{A}$ be a projection (that is, $p^{2}=p$ ), and consider the complex Banach algebra $p \mathcal{A} p$ which has unit $p$. The exponential function in $p \mathcal{A} p$ will be denoted by $\exp _{p}$. Now, let $E$ be a real normed space, let $A: E \rightarrow p \mathcal{A} p$ be a continuous and linear mapping (here $p \mathcal{A} p$ is considered as a real vector space), and set

$$
f(x):=\exp _{p}(A(x))=p \exp (A(x)) p \quad(x \in E) .
$$

As an immediate consequence of (1) the function $f$ is a continuous solution of the functional equation

$$
f(x+y)=\lim _{n \rightarrow \infty}(f(x / n) f(y / n))^{n} \quad(x, y \in E) .
$$

In this paper we prove conversely that all continuous solutions of (3) are of type (2). More precisely we have

Theorem 1. Let $f: E \rightarrow \mathcal{A}$ be a continuous function which satisfies (3). Then $p=f(0)$ is a projection, and there exists a unique continuous linear mapping $A: E \rightarrow p \mathcal{A} p$ such that

$$
f(x)=\exp _{p}(A(x)) \quad(x \in E) .
$$

2000 Mathematics Subject Classification: 39B52, 46H99.

Key words and phrases: Lie's product formula, functional equation, Banach algebras. 
In the proof of Theorem 1 we use the following proposition. Note that $\sigma(a)$ denotes the spectrum of $a \in \mathcal{A}$.

Proposition 1. Let $a, b \in \mathcal{A}$.

(i) If $a^{3}=a$ and $\sigma(a) \subseteq\{0,1\}$, then $a^{2}=a$.

(ii) If $\exp (a)=\exp (b)$ and $\|a\|<\pi$, then $a b=b a$.

(iii) If $\exp (t a)=\mathbf{1}(t>0)$, then $a=0$.

Proof. (i) and (ii) follow from [2, Propositions 8.11 and 18.12], respectively, and (iii) follows by differentiation.

Proof of Theorem 1.

SteP 1. We have

$$
p^{2}=p
$$

Proof. From (3) we obtain

$$
f(0)=\lim _{n \rightarrow \infty} f(0)^{2 n} \Rightarrow f(0)^{3}=\lim _{n \rightarrow \infty} f(0)^{2 n+2}=f(0) .
$$

Hence $\sigma(f(0)) \subseteq\{-1,0,1\}$. Now, assume $-1 \in \sigma(f(0))$. Choose open sets $U, V \subseteq \mathbb{C}$ such that $U \cap V=\emptyset,-1 \in U$, and $0,1 \in V$. Then $\sigma(f(0)) \subseteq U \cup V$ and $\sigma(f(0)) \cap U \neq \emptyset$. Since $f(0)^{2 n} \rightarrow f(0)$ as $n \rightarrow \infty$, Theorem 3.4.4 in [1] proves

$$
\sigma\left(f(0)^{2 n}\right) \cap U \neq \emptyset
$$

for $n$ sufficiently large. But $\sigma\left(f(0)^{2 n}\right) \subseteq\{0,1\} \subseteq V$, a contradiction. Therefore $\sigma(f(0)) \subseteq\{0,1\}$ and (4) follows from Proposition 1(i).

Step 2. We have

$$
f(x)=p f(x)=f(x) p=p f(x) p \quad(x \in E) ;
$$

in particular $f(x) \in p \mathcal{A} p(x \in E)$.

Proof. According to (3),

$$
\begin{aligned}
p f(x) & =f(0) f(x+0)=f(0) \lim _{n \rightarrow \infty}(f(x / n) f(0))^{n} \\
& =\left(\lim _{n \rightarrow \infty}(f(0) f(x / n))^{n}\right) f(0)=f(0+x) f(0)=f(x) p
\end{aligned}
$$

for each $x \in E$. Thus,

$$
\begin{aligned}
f(x) & =f(x+0)=\lim _{n \rightarrow \infty} f(x / n)^{n} f(0)^{n} \stackrel{(4)}{=} \lim _{n \rightarrow \infty} f(x / n)^{n} f(0)^{n+1} \\
& =\left(\lim _{n \rightarrow \infty} f(x / n)^{n} f(0)^{n}\right) f(0)=f(x+0) f(0)=f(x) p,
\end{aligned}
$$

and we have (5). In particular, if $p=0$ then $f(x)=0(x \in E)$.

SteP 3. For $x \in E$ and $m \in \mathbb{N}$,

$$
f(m x)=f(x)^{m} .
$$


Proof. Note that by (5),

$$
f(x)=\lim _{n \rightarrow \infty} f(x / n)^{n} \quad(x \in E) .
$$

First consider $m=2$. Again from (3) we obtain

$$
f(2 x)=f(x+x)=\lim _{n \rightarrow \infty} f(x / n)^{2 n}=\lim _{n \rightarrow \infty}\left(f(x / n)^{n}\right)^{2}=f(x)^{2} .
$$

Now, let $m>2$ and suppose $f(m x)=f(x)^{m}(x \in E)$. Then

$$
\begin{aligned}
f((m+1) x) & =\lim _{n \rightarrow \infty}(f(m x / n) f(x / n))^{n}=\lim _{n \rightarrow \infty}\left(f(x / n)^{m} f(x / n)\right)^{n} \\
& =\lim _{n \rightarrow \infty}\left(f(x / n)^{n}\right)^{m+1}=f(x)^{m+1} .
\end{aligned}
$$

Thus (6) holds by induction.

Next, for each $x \in E$ let $f_{x}: \mathbb{R} \rightarrow \mathcal{A}$ be defined by

$$
f_{x}(\alpha):=f(\alpha x) \text {. }
$$

STEP 4. We have

$$
f_{x}(\alpha) f_{x}(\beta)=f_{x}(\beta) f_{x}(\alpha) \quad(\alpha, \beta \geq 0, x \in E) .
$$

Proof. Let $x \in E$ and $m, n, r, s \in \mathbb{N}$. Now

$$
\begin{gathered}
f_{x}(1 / r) f_{x}(1 / s)=f(x / r) f(x / s)=f\left(s \frac{x}{r s}\right) f\left(r \frac{x}{r s}\right) \\
\stackrel{(6)}{=} f\left(\frac{x}{r s}\right)^{s+r}=f_{x}(1 / s) f_{x}(1 / r) .
\end{gathered}
$$

Hence

$$
\begin{aligned}
f_{x}(m / r) f_{x}(n / s) & =f\left(m \frac{x}{r}\right) f\left(n \frac{x}{s}\right) \stackrel{(6)}{=} f(x / r)^{m} f(x / s)^{n}=f(x / s)^{n} f(x / r)^{m} \\
& \stackrel{(6)}{=} f\left(n \frac{x}{s}\right) f\left(m \frac{x}{r}\right)=f_{x}(n / s) f_{x}(m / r) .
\end{aligned}
$$

Therefore (7) is valid for $\alpha, \beta \in \mathbb{Q} \cap[0, \infty)$, hence for $\alpha, \beta \in[0, \infty)$, since $f$ is continuous.

SteP 5. We have

$$
f_{x}(\alpha+\beta)=f_{x}(\alpha) f_{x}(\beta) \quad(\alpha, \beta \geq 0, x \in E) .
$$

Proof. For $\alpha, \beta \geq 0$,

$$
\begin{aligned}
f_{x}(\alpha+\beta) & =f(\alpha x+\beta x) \stackrel{(3)}{=} \lim _{n \rightarrow \infty}\left(f\left(\alpha \frac{x}{n}\right) f\left(\beta \frac{x}{n}\right)\right)^{n} \\
& \stackrel{(7)}{=} \lim _{n \rightarrow \infty} f\left(\alpha \frac{x}{n}\right)^{n} f\left(\beta \frac{x}{n}\right)^{n} \stackrel{(6)}{=} \lim _{n \rightarrow \infty} f(\alpha x) f(\beta x)=f_{x}(\alpha) f_{x}(\beta) .
\end{aligned}
$$

Step 6. The limit

$$
A(x):=\lim _{\alpha \rightarrow 0+} \frac{1}{\alpha}\left(f_{x}(\alpha)-p\right)
$$


exists for each $x \in E$. Moreover

$$
A(x) \in p \mathcal{A} p \quad(x \in E)
$$

and

$$
f(\alpha x)=p+\sum_{n=1}^{\infty} \frac{\alpha^{n}}{n !} A(x)^{n} \quad(\alpha \geq 0) .
$$

Note that in particular for $\alpha=1$ we have

$$
f(x)=p+\sum_{n=1}^{\infty} \frac{A(x)^{n}}{n !}=\exp _{p}(A(x))=p \exp (A(x)) p \quad(x \in E) .
$$

Proof. Since $f_{x}:[0, \infty) \rightarrow \mathcal{A}$ is a continuous solution of the functional equation in (8), the existence of the limit in (9) and the equation (10) follow from [3, Theorem 9.4.2]. Now, (10) follows from (5) and (9).

STEP 7. We have

$$
A(\beta x)=\beta A(x) \quad(\beta \geq 0, x \in E) .
$$

Proof. Obviously (13) holds for $\beta=0$. For $\beta>0$,

$$
A(\beta x)=\lim _{\alpha \rightarrow 0+} \frac{1}{\alpha}(f(\alpha \beta x)-p)=\lim _{\alpha \rightarrow 0+} \frac{\beta}{\alpha \beta}\left(f_{x}(\alpha \beta)-p\right)=\beta A(x) .
$$

STEP 8. For $x, y \in E$,

$$
\exp _{p}(A(x+y))=\exp _{p}(A(x)+A(y))
$$

and

$$
A(x+y)(A(x)+A(y))=(A(x)+A(y)) A(x+y) .
$$

Proof. Fix $x, y \in E$ and let $\alpha>0$. Set

$$
a:=A(\alpha(x+y)), \quad b:=A(\alpha x)+A(\alpha y) .
$$

Then, by Lie's product formula, and by (12) and (13),

$$
\begin{aligned}
\exp _{p}(b) & =\lim _{n \rightarrow \infty}\left(\exp _{p}(A(\alpha x / n)) \exp _{p}(A(\alpha y / n))\right)^{n} \\
& \stackrel{(3)}{=} \lim _{n \rightarrow \infty}(f(\alpha x / n) f(\alpha y / n))^{n}=f(\alpha x+\alpha y) \\
& =\exp _{p}(A(\alpha x+\alpha y))=\exp _{p}(a) .
\end{aligned}
$$

For $\alpha=1$ we obtain (14), and by choosing $\alpha>0$ such that

$$
\|a\|=\alpha\|A(x+y)\|<\pi,
$$

Proposition 1(ii) proves $a b=b a$, hence (15).

SteP 9. We have

$$
A(x+y)=A(x)+A(y) \quad(x, y \in E) .
$$


Proof. According to (14) and (15) we have

$$
\exp _{p}(A(x+y)-(A(x)+A(y)))=p \quad(x, y \in E) .
$$

Fix $x, y \in E$. By (13),

$$
\exp _{p}(t(A(x+y)-(A(x)+A(y))))=p \quad(t>0),
$$

and Proposition 1(iii) proves (16).

SteP 10. We have

$$
A(\alpha x)=\alpha A(x) \quad(\alpha \in \mathbb{R}, x \in E) .
$$

Proof. Fix $x \in E$. Then

$\exp _{p}(t(A(x)+A(-x))) \stackrel{(13)}{=} \exp _{p}(A(t x)+A(-t x)) \stackrel{(16)}{=} \exp _{p}(A(t x-t x))=p$ for each $t>0$. Again, $A(-x)=-A(x)$ follows from Proposition 1(iii). In combination with (13) this gives (17).

SteP 11. The linear mapping $A: E \rightarrow p \mathcal{A} p$ is continuous.

Proof. It is sufficient to prove that $A$ is continuous at 0 . Assume the contrary. Then there is a sequence $\left(x_{n}\right)$ in $E$ with $\left\|x_{n}\right\|=1(n \in \mathbb{N})$ and $\left\|A\left(x_{n}\right)\right\| \rightarrow \infty(n \rightarrow \infty)$. Set

We have

$$
y_{n}=\frac{x_{n}}{3\left\|A\left(x_{n}\right)\right\|}, \quad z_{n}=\frac{A\left(x_{n}\right)}{3\left\|A\left(x_{n}\right)\right\|} .
$$

$$
f\left(y_{n}\right)=\exp _{p}\left(A\left(y_{n}\right)\right)=\exp _{p}\left(z_{n}\right) \rightarrow p \quad(n \rightarrow \infty),
$$

because $y_{n} \rightarrow 0(n \rightarrow \infty)$. Since $\left\|\exp _{p}\left(z_{n}\right)-p\right\| \leq 1 / 2<1$, we conclude

$$
z_{n}=\log _{p}\left(\exp _{p}\left(z_{n}\right)\right) \rightarrow 0 \quad(n \rightarrow \infty)
$$

a contradiction. Here $\log _{p}$ denotes the power series

$$
\log _{p}(p+a)=\sum_{k=1}^{\infty} \frac{(-1)^{k+1}}{k} a^{k} \quad(a \in p \mathcal{A} p,\|a\|<1) .
$$

Finally, concerning the uniqueness of $A$, let $B: E \rightarrow p \mathcal{A} p$ be a continuous linear operator such that

$$
f(x)=\exp _{p}(B(x)) \quad(x \in E) .
$$

Then

$$
\frac{1}{\alpha}(f(\alpha x)-p)=\frac{1}{\alpha}\left(\exp _{p}(\alpha(B(x)))-p\right) \rightarrow B(x) \quad(\alpha \rightarrow 0+) .
$$

According to $(9), A(x)=B(x)(x \in E)$.

As an application of Theorem 1 we may characterize in terms of $A$ those continuous solutions of (3) which satisfiy the exponential equation of Cauchy

$$
f(x+y)=f(x) f(y) \quad(x, y \in E) .
$$


Corollary 1. Let $f: E \rightarrow \mathcal{A}$ be a continuous solution of (3), and let $p$ and $A: E \rightarrow p \mathcal{A} p$ be as in Theorem 1. Then (18) holds if and only if

$$
A(x) A(y)=A(y) A(x) \quad(x, y \in E) .
$$

Proof. If (19) holds then clearly

$$
f(x+y)=\exp _{p}(A(x)+A(y))=\exp _{p}(A(x)) \exp _{p}(A(y))=f(x) f(y)
$$

for $x, y \in E$.

Now, let (18) be valid. Then $f(x) f(y)=f(y) f(x)$, hence

$$
\exp _{p}(A(x)) \exp _{p}(A(y))=\exp _{p}(A(y)) \exp _{p}(A(x)) \quad(x, y \in E) .
$$

Fix $x, y \in E$ and let $\alpha>0$ be such that

$$
\max \{\|A(\alpha x)\|,\|A(\alpha y)\|\}<\pi .
$$

According to the result in [5],

$$
A(\alpha x) A(\alpha y)=A(\alpha y) A(\alpha x),
$$

from which (19) follows.

Remark. Theorem 1 is also valid if $\mathcal{A}$ is a real Banach algebra with unit 1 . In this case apply the complex version to the complexification $\mathcal{A}_{\mathbb{C}}$ of $\mathcal{A}$ and note that $p=f(0) \in \mathcal{A}$ and that $A$ maps $E$ to $p \mathcal{A} p$ according to $(9)$.

As an example consider $E=\mathbb{R}$ and assume that $f: \mathbb{R} \rightarrow \mathcal{A}$ is a continuous solution of (3) with $f(0)$ invertible. Then by Theorem $1, f(0)=\mathbf{1}$ and there is a unique $a \in \mathcal{A}$ such that

$$
f(x)=\exp (x a) \quad(x \in \mathbb{R}) .
$$

Here $f$ is a solution of (18).

On the other hand consider $E=\mathbb{R}^{2}$ and again assume that $f: \mathbb{R}^{2} \rightarrow \mathcal{A}$ is a continuous solution of (3) with $f(0)$ invertible. Then there exist unique $a, b \in \mathcal{A}$ such that

$$
f\left(\left(x_{1}, x_{2}\right)\right)=\exp \left(x_{1} a+x_{2} b\right) \quad\left(\left(x_{1}, x_{2}\right) \in \mathbb{R}^{2}\right) .
$$

Here, $f$ is a solution of (18) if and only if $a b=b a$.

\section{References}

[1] B. Aupetit, A Primer on Spectral Theory, Universitext, Springer, New York, 1991.

[2] F. F. Bonsall and J. Duncan, Complete Normed Algebras, Ergeb. Math. Grenzgeb. 80, Springer, Berlin, 1973.

[3] E. Hille and R. S. Phillips, Functional Analysis and Semigroups, Amer. Math. Soc. Colloq. Publ. 31, Amer. Math. Soc., Providence, RI, 1957.

[4] M. Reed and B. Simon, Methods of Modern Mathematical Physics. I. Functional Analysis, 2nd ed., Academic Press, New York, 1980. 
[5] C. Schmoeger, Remarks on commuting exponentials in Banach algebras, Proc. Amer. Math. Soc. 127 (1999), 1337-1338.

[6] H. F. Trotter, On the product of semi-groups of operators, ibid. 10 (1959), 545-551.

Mathematisches Institut I

Universität Karlsruhe

D-76128 Karlsruhe, Germany

E-mail: Gerd.Herzog@math.uni-karlsruhe.de

Christoph.Schmoeger@math.uni-karlsruhe.de

Received August 28, 2005

Revised version June 8, 2006 